\title{
MONOTONE REAL ANALYTIC MAPS WHICH ARE NOT HOMEOMORPHISMS
}

\author{
A. C. HSU AND J. G. TIMOURIAN 1
}

ABSTRACT. Monotone, proper, real analytic, onto maps which have nonempty branch sets are exhibited. The smash product maps are shown to belong to this class of maps. Maps with branch set a submanifold and with discrete branch set images are classified (locally).

1. Introduction. In [2, p. 178] P. T. Church asks whether there exist any monotone proper real analytic onto maps $f: M^{n} \rightarrow N^{n}$ which are not homeomorphisms. Under additional hypotheses, such as requiring $f$ to be $Z_{2}$-acyclic, $\operatorname{dim} B_{f}<n / 2, M^{n}=S^{n}$, or $M^{n}$ closed and $M^{n}=N^{n}$, $f$ must be a homeomorphism. In this note we give examples which demonstrate that $f$ need not be a homeomorphism (see $\$ \$ 3$ and 5) and give information (in $\$ 6$ ) about the local structure of differentiable maps related to the examples. Definitions, conventions, and related results can be found in the survey article by Church [2].

2. Examples. In each of these $f$ is a proper continuous function such that the branch set $B_{f}$, the set of points at which $f$ fails to be a local homeomorphism, is a submanifold of the domain and $f\left(B_{f}\right)$ is a single point.

(i) $f: R^{1} \rightarrow R^{1}$ defined by $f(x)=x^{2}$,

(ii) $f: R^{2} \rightarrow R^{2}$ defined by $f(z)=z^{m}$, where we are considering $R^{2}$ to be the space of complex numbers and $m$ is an integer $>1$,

(iii) $f: M^{n} \rightarrow R^{n}$ defined by $f(x)=0$, where $M^{n}$ is compact,

(iv) $f: M^{2} \rightarrow R^{2}$, where $M^{2}$ is the (open) Möbius band, and $f$ is defined by the natural map from $M^{2}$ to the quotient space obtained by identifying the central circle to a point,

(v) the composition of the maps in (iv) and (ii),

(vi) maps more general than the one in (iv) which are constructed by letting $M^{n}$ be the open mapping cylinder of one of these bundle maps: $n>1$, $h: S^{n-1} \rightarrow B^{n-1}$ with fiber $S^{0}$, where $B^{n-1}$ is homotopically equivalent

Received by the editors February 2, 1974.

AMS (MOS) subject classifications (1970). Primary 54C10; Secondary 57D45. Key words and phrases. Monotone, open, branch set.

1Supported by NRC of Canada Grant A7357. 
to $R P^{n-1}$ (examples are the covering maps from $S^{n-1}$ to $R P^{n-1}$ );n>3 even, $h: S^{n-1} \rightarrow B^{n-2}$ with fiber $S^{1}$, where $B^{n-2}$ is homotopically equivalent to $C P^{n / 2-1}$ (examples are the Hopf maps from $S^{n-1}$ to $C P^{n / 2-1}$ ); $n>5$ divisible by $4, h: S^{n-1} \rightarrow B^{n-4}$ with fiber $S^{3}$ (examples are the Hopf maps from $S^{n-1}$ to $Q P^{n / 4-1}$ ); and $h: S^{15} \rightarrow S^{8}$ with fiber $S^{7}$ (an example is the Hopf map). Let $f: M^{n} \rightarrow R^{n}$ be defined by the natural map from $M^{n}$ to the quotient space obtained by identifying the image of $h$ to a point.

3. Real analytic examples. Of the maps in $\$ 2$, (i) and (ii) are real analytic, as is the map in (iii) if $M^{n}$ is a real analytic manifold. The map in (iv) is a special case of the maps in (vi), when $h: S^{1} \rightarrow R P^{1}$. If, in (vi), $h$ is selected to be a differentiable map with maximal rank, then the mapping cylinder of $h$ can be made into a differentiable manifold, and the resulting map $f$ will be differentiable except possibly at the points in $B_{f}$. By $[3$, p. 95, (3.3)] $f$ can be followed by a homeomorphism so that the composition of the two maps is differentiable.

To construct real analytic examples which are topologically equivalent to the maps in (vi), let $H$ denote either the real numbers, complex numbers, or the quaternions, and let $k$ denote the real dimension of $H$. Let $H_{m}$ $(m \geq 3)$ be the right vector space whose elements are ordered sets of $m$ elements of $H$ and let $S^{k m-1}$ be the unit sphere in $H_{m}$. Define a map $\alpha: H_{m} \rightarrow H_{m(m-1) / 2} \times R^{1}$ by

$$
\alpha\left(x_{1}, x_{2}, \ldots, x_{m}\right)=\left(x_{1} \bar{x}_{2}, \ldots, x_{1} \bar{x}_{m}, x_{2} \bar{x}_{3}, \ldots, x_{m-1} \bar{x}_{m}, \sum_{i=1}^{m} i x_{i} \bar{x}_{i}\right) .
$$

The map $\alpha$ is real analytic, its restriction $\beta$ to $s^{k m-1}$ is real analytic. Note that $\beta$ maps every $(k-1)$-sphere $\left\{\left(\lambda x_{1}, \ldots, \lambda x_{m}\right) ; \lambda \in H,|\lambda|=1\right\}$ to a single point, and $\beta\left(S^{k m-1}\right)$ is the projective space associated with $H_{m}$ embedded real analytically in $H_{m(m-1) / 2} \times R^{1}$ (the map $\beta$ is the Hopf map). Consider the image $M^{k(m-1)}$ of the region $U=\left\{\left(x_{1}, x_{2}, \ldots, x_{m}\right) \epsilon\right.$ $\left.S^{k m-1} ;\left|x_{1}\right|<1 / 2\right\}$ under $\beta$. Then $M^{k(m-1)}$ is a real analytic $k(m-1)$-manifold. Let $N^{k(m-1)}$ be the open $k(m-1)$-disk of radius $\sqrt{ } 3 / 4$ centered at the origin and contained in $R^{k(m-1)}$. Define $f: M^{k(m-1)} \rightarrow N^{k(m-1)}$ to be the restriction of the projection map of $H_{m(m-1) / 2} \times R$ onto the first $k(m-1)$ (real) coordinates:

$$
f\left(x_{1} \bar{x}_{2}, \ldots, x_{m-1} \bar{x}_{m}, \sum_{i=1}^{m} i x_{i} \bar{x}_{i}\right)=\left(x_{1} \bar{x}_{2}, \ldots, x_{1} \bar{x}_{m}\right) .
$$

Then $f$ is the desired analytic example. 
4. Remarks. To clarify why the examples in $\$ 3$ have the properties claimed we will examine in more detail the case in which $H=$ real numbers. In this case the map a restricted to the unit sphere $S^{m-1}$ has constant rank equal to $m-1$, takes antipodal points to the same image, and is in fact a 2-to-1 covering map onto its image. Thus $a\left(S^{m-1}\right)$ is indeed an analytically embedded real projective $(m-1)$-plane. If $\pi$ is the projection map of $R^{m(m-1) / 2} \times R$ to the first $m-1$ coordinates, then $\alpha^{-1}\left(\pi^{-1}(0) \cap \alpha\left(S^{m-1}\right)\right)$ is the union of the equator $(m-2)$-sphere $S^{m-2}$ in $S^{m-1}$ defined by $x_{1}=0$, and the North and South poles of $s^{m-1}$. By considering only the region $U$ consisting of those points with $\left|x_{1}\right|<1 / 2$ we are confining ourselves to a neighborhood of $S^{m-2}$, so that $\alpha^{-1}\left(\pi^{-1}(0) \cap \alpha(U)\right)=S^{m-2}$. The image of $U$ under $\alpha$ will be a manifold $M^{m-1}$ which is a neighborhood of $\alpha\left(S^{m-2}\right)=$ real projective $(m-2)$-space. Note that on each component of $U-S^{m-2}$ the map $\alpha$ is a diffeomorphism (to its image). Each $(m-2)$-sphere defined by $x_{1}=$ constant, $0<\left|x_{1}\right|<1 / 2$, is mapped to an $(m-2)$-sphere in $M^{m-1}$ and then by $\pi$ (diffeomorphically) to an $(m-2)$-sphere of constant radius (depending on $\left|x_{1}\right|$ ) in $R^{m-1}$. The disk in $R^{m-1}$ is selected to have radius $\sqrt{ } 3 / 4$ in order to make the desired map onto. The image of $U$ under $\alpha$, that is, $M^{m-1}$, can be identified with the mapping cylinder of the standard covering map from $S^{m-2}$ to real projective $(m-2)$-space.

Similar arguments demonstrate the examples in the other situations. In each case $B_{f}$ turns out to be the appropriate projective space corresponding to the equatorial sphere in $s^{k m-1}$ defined by $\left|x_{1}\right|=0$. The maps are monotone, onto, and real analytic. Together with the examples in $\$ 5$ they represent the counterexamples mentioned in the first paragraph. These examples also demonstrate that the condition " $\operatorname{dim} B_{f}<n / 2$ " in $[4, \mathrm{p} .33,(6.2)]$ is best possible.

To define examples on the closed manifolds $\beta\left(S^{k m-1}\right)=H$ projective $(m-1)$-space let

$$
\bar{f}\left(x_{1} \bar{x}_{2}, \ldots, x_{m-1} \bar{x}_{m}, \sum_{i=1}^{m} i x_{i} \bar{x}_{i}\right)=\left(2 x_{1} \bar{x}_{2}, \ldots, 2 x_{1} \bar{x}_{m}, 1-2 x_{1} \bar{x}_{1}\right) .
$$

Then $\bar{f}: \beta\left(S^{k m-1}\right) \rightarrow S^{k(m-1)}$ and the branch set is again the $H$ projective $(m-2)$-space corresponding to the sphere in $S^{k m-1}$ defined by $\left|x_{1}\right|=0$.

5. Smash product examples. The Theorem in $\$ 6$ will demonstrate that the examples in $\$ 2$ in a sense exhaust the (differentiable) possibilities for maps with $B_{f}$ a submanifold and $f\left(B_{f}\right)$ a single point. Examples can 
also be constructed in which $B_{f}$ is not a submanifold. The smash product maps from $S^{n} \times S^{p}$ to $S^{n+p}$ which are defined by collapsing a one point union of $S^{n}$ and $S^{p}$ in $S^{n} \times S^{p}$ to a point are monotone $(n, p>0)$, onto, and have $B_{f}=S^{n} \vee S^{p}$. These maps can also be made differentiable by [3, p. 95, (3.3)]. In fact the specific question posed in [2] and [4] was whether these maps could be done real analytically. We will show here that the answer to this question is yes. Note that these examples are between closed manifolds.

Define $\alpha: R^{n+1} \times R^{q+1} \rightarrow R^{n+q+1}$ by $a\left(x_{1}, \ldots, x_{n+1}, y_{1}, \ldots, y_{q+1}\right)=\left(x_{1} y_{1}, \ldots, x_{n+1} y_{1}, x_{1} y_{2}, \ldots, x_{1} y_{q+1}\right)$. Then an analytic copy of $S^{n} \times S^{q} \subset R^{n+1} \times R^{q+1}$ defined by

$$
\left\{\begin{array}{l}
\left(x_{1}-1\right)^{2}+x_{2}^{2}+\cdots+x_{n+1}^{2}=1 \\
\left(y_{1}-1\right)^{2}+y_{2}^{2}+\cdots+y_{q+1}^{2}=1
\end{array}\right\}
$$

is mapped to a compact set $S \subset R^{n+q+1}$.

Let $A \subset R^{n+q+1}$ be defined by $z=\left(z_{1}, z_{2}, \ldots, z_{n+q+1}\right) \in A$ if either $z=0$ or $z_{1} \neq 0$ and

$$
F(z)=4 z_{1}^{3}-\left(z_{1}^{2}+z_{2}^{2}+\cdots+z_{n+1}^{2}\right)\left(z_{1}^{2}+z_{n+2}^{2}+\cdots+z_{n+q+1}^{2}\right)=0 .
$$

Then $S \subset A$ and, in addition, given any $z \in A$, either $z=0 \in S$, or $z_{1} \neq 0$ and $\alpha(x, y)=z$, where

and

$$
x_{1}=\frac{2 z_{1}^{2}}{z_{1}^{2}+z_{2}^{2}+\cdots+z_{n+1}^{2}}, \quad x_{i}=\frac{z_{i} x_{i}}{z_{1}}, \quad i=1,2, \ldots, n+1,
$$

$$
y_{1}=\frac{2 z_{1}^{2}}{z_{1}^{2}+z_{n+2}^{2}+\cdots \cdot+z_{n+q+1}^{2}}, \quad y_{i}=\frac{z_{n+i} y_{1}}{z_{1}}, \quad i=2,3, \ldots, q+1 .
$$

Since $(x, y)$ is an element of our domain torus, $A=S$.

If $p=(1,0, \ldots, 0)$ and $z \in S, z_{1} \neq 0$, then

$$
\begin{aligned}
(z-p) \cdot \operatorname{grad} F & =\left(\left(z_{1}-1\right), z_{2}, \ldots, z_{n+q+1}\right) \cdot\left(\frac{\partial F}{\partial z_{1}}, \frac{\partial F}{\partial z_{2}}, \ldots, \frac{\partial F}{\partial z_{n+q+1}}\right) \\
& =-12 z_{1}^{2}+2 z_{1}\left(z_{2}^{2}+z_{3}^{2}+\cdots+z_{n+q+1}^{2}\right) .
\end{aligned}
$$

This last expression is zero if and only if $x_{1}+y_{1}-x_{1} y_{1}-3=0$, and such cannot occur for $0<x_{1}, y_{1} \leq 2$. Thus any line through the points $z \in S$, 
$z \neq 0$, and $p$ intersects $S$ transversely. For example, the line through the point $z$ defined by $z_{1}=z_{2}=z_{n+2}=1, z_{i}=0$ otherwise, and $p$, intersects $S$ transversely in precisely two points. The $z_{1}$ axis intersects $S$ at $z=0$ and $z=(4,0, \ldots, 0)$, so we can conclude that any line through $p$ intersects $S$ in precisely two points, and the open segment joining them contains $p$ and is in the interior of the region bounded by $S$. By composing the appropriate restriction of $a$ with a normalization of $S$ about $p$ we get a real analytic map $\lambda$ from $S^{n} \times S^{p}$ to $S^{n+q}$ (a unit sphere in $R^{n+q+1}$ centered at $p$ ) which is the smash product map: $\lambda: S^{n} \times S^{p} \rightarrow S^{n+q}$ defined by $\lambda(x, y)=\frac{\left(x_{1} y_{1}-1, x_{2} y_{1}, \ldots, x_{n+1} y_{1}, x_{1} y_{2}, x_{1} y_{3}, \ldots, x_{1} y_{q+1}\right)}{\left(\left(x_{1} y_{1}-1\right)^{2}+y_{1}^{2}\left(x_{2}^{2}+x_{3}^{2}+\cdots+x_{n+1}^{2}\right)+x_{1}^{2}\left(y_{2}^{2}+y_{3}^{2}+\cdots+y_{q+1}^{2}\right)\right)^{1 / 2}}$.

6. Theorem. Let $f: M^{n} \rightarrow N^{n}$ be a proper differentiable map with $f\left(B_{f}\right)$ discrete and $f^{-1}(y)$ a (smooth) submanifold of $M^{n}$ for each $y \in N^{n}$. Then for each $x \in B$, and component $B^{k}$ of $f^{-1}(f(x))$ containing $x$, there exist neighborhoods $U$ of $B^{k}$ and $V$ of $f(x)$ such that $f \mid U: U \rightarrow V$ is topologically equivalent to one of the examples in $\$ 2$.

Proof. Choose neighborhoods $V$ of $f(x)$ and $U$ of $B^{k}$ such that $V$ is a coordinate neighborhood, $U$ is a component of $f^{-1}(V)$, and $U \cap\left(f^{-1}(f(x))\right)$ $=U \cap B_{f}=B^{k}$. If $k=n$, then $B^{n}=U$ is a component of $M^{n}$, so $f$ on this component is topologically equivalent to the constant map in $\$ 2$ (iii). So assume $k<n$. Since the branch set of $f \mid U-B^{k}$ is empty, $f \mid U-B^{k}: U-$ $B^{k} \rightarrow V-f(x)$ is a $d$-to- 1 covering map. When $n=1$ this implies that $d=$ 2 and $f \mid U$ is topologically equivalent to the map in $\$ 2$ (i). When $n \geq 3$, $V-f(x)$ is simply connected, so $f \mid U-B^{k}$ is a homeomorphism. When $n=$ 2 , let $h g$ be a factorization of $f \mid U$ into a monotone map followed by a light map with at most one point in its branch set. If $B^{k}$ consists of a single point, then $g$ is a homeomorphism. Thus $h \mid g(U)-g\left(B^{k}\right)$ is a $d$-to-1 covering map onto $V-f(x)$, which implies that $f \mid U$ is topologically equivalent to an example from $\$ 2$ (ii). If $B^{k}$ is not a single point, then either $f \mid U-$ $B^{k}$ is a homeomorphism, or $g \mid U-B^{k}$ is a homeomorphism and $h$ is topologically equivalent to an example from $\$ 2$ (ii). Thus we may confine our attention to situations in which $\operatorname{dim} B^{k}<n, n \geq 2$, and $f \mid U$ is a monotone map (when $n=2$ and $B^{k}$ is not a single point, we will show that the monotone map $g$ must be topologically equivalent to the example in $\$ 2$ (iv), and thus $f \mid U$ is topologically equivalent to the example in $\S 2((\mathrm{iv})$ or (v))).

Let $\xi$ be the disk bundle obtained by restricting the fibers of the normal bundle of $B^{k}$ to vectors with norm $\leq 1$. Let $W \subset M^{n}$ be the set in a 
tubular neighborhood of $B^{k}$ which corresponds to $\xi$. Since $\operatorname{dim} B^{k}=k, \partial W$ is the total space of a bundle over $B^{k}$ with fiber $S^{n-k-1}$, and $W-B^{k}$ is the total space of a bundle over $B^{k}$ with fiber $S^{n-k-1} \times[0,1)$. Thus $W-B^{k}$ is homeomorphic to $\partial W \times[0,1)$. Since $f \mid W-B^{k}$ is a homeomorphism, and $f\left(\right.$ int $\left.W-B^{k} \cup\{f(x)\}\right)$ is a neighborhood of $f(x)$ in $V$, the open cone of $f(\partial W)$ is a manifold. Thus $f(\partial W)$ (and hence $\partial W)$ is an $(n-1)$-sphere [6, p. $141,(4.2)]$. The neighborhood $W$ of $B^{k}$ is the mapping cylinder of the bundle $h: \partial W \rightarrow B^{k}$ with fiber $S^{n-k-1}$.

If $n \neq 4$ or 5 , then $n-k-1=0$, the fiber is $S^{0}$, and $B^{k}$ is homotopically equivalent to $R P^{n-1}$, or by $[1, \mathrm{p} .353,(5.1)]$ : the fiber $S^{n-k-1}$ is $S^{1}, S^{3}$ or $S^{7}$; if the fiber is $S^{1}, B^{k}$ is homotopically equivalent to $C P^{n / 2-1}$; if the fiber is $S^{7}, B^{k}$ (in our situation) must be $S^{8}$, and thus, $\partial W=S^{15}$ (see the argument in $[5$, p. $64,(2.7)]$ ). If the fiber is $S^{3}$, the Gysin sequence implies that $n$ must be divisible by 4 . Choose $U$ and $V$ so that $U=W, V=$ $f(W)$. Then $f \mid U$ is topologically equivalent to one of the examples in $\$ 2$.

\section{REFERENCES}

1. W. Browder, Higher torsion in H-spaces, Trans. Amer. Math. Soc. 108 (1963), 353-375. MR 27 \#5260.

2. P. T. Church, Differentiable monotone mappings and open mappings, Proc. First Conf. on Monotone Mappings and Open Mappings (SUNY at Binghamton, Binghamton, N. Y., 1970), State Univ. of New York at Binghamton, Binghamton, N. Y., 1971, pp. 145-183. MR 43 \#6938.

3. - Differentiable open maps on manifolds, Trans. Amer. Math. Soc. 109 (1963), 87-100. MR 27 \# 4245.

4. P. T. Church and W. D. Nathan, Real analytic maps on manifolds, J. Math. Mech. 19 (1969/70), 19-36. MR 39 \# 4860.

5. J. G. Timourian, Fiber bundles with discrete singular set, J. Math. Mech. $18(1968 / 69), 61-70$. MR 38 \#3875.

6. A. Wallace, Local cone structures on varieties, Indiana Univ. Math. J. 24 (1974), 131-141.

DEP ARTMENT OF MATHEMATICS, UNIVERSITY OF ALBERTA, EDMONTON, ALBERTA, CANADA (Current address of J.G. Timourian) 